\title{
Intrahemispheric cortico-cortical connections of the human auditory cortex
}

\author{
Leila Cammoun · Jean Philippe Thiran • \\ Alessandra Griffa • Reto Meuli • Patric Hagmann • \\ Stephanie Clarke
}

Received: 23 March 2014/ Accepted: 6 August 2014/Published online: 31 August 2014

(C) Springer-Verlag Berlin Heidelberg 2014

\begin{abstract}
The human auditory cortex comprises the supratemporal plane and large parts of the temporal and parietal convexities. We have investigated the relevant intrahemispheric cortico-cortical connections using in vivo DSI tractography combined with landmark-based registration, automatic cortical parcellation and whole-brain structural connection matrices in 20 right-handed male subjects. On the supratemporal plane, the pattern of connectivity was related to the architectonically defined earlystage auditory areas. It revealed a three-tier architecture characterized by a cascade of connections from the primary auditory cortex to six adjacent non-primary areas and from there to the superior temporal gyrus. Graph theory-driven analysis confirmed the cascade-like connectivity pattern and demonstrated a strong degree of segregation and hierarchy within early-stage auditory areas. Putative higher-order areas on the temporal and parietal convexities had more widely spread local connectivity and long-range connections with the prefrontal cortex; analysis of optimal community structure revealed five distinct modules in each hemisphere. The pattern of temporo-parieto-frontal connectivity was partially asymmetrical. In conclusion, the
\end{abstract}

L. Cammoun $(\bowtie) \cdot$ S. Clarke $(\bowtie)$

Service de Neuropsychologie et de Neuroréhabilitation, CHUV,

Université de Lausanne, Lausanne, Switzerland

e-mail: Leila.Cammoun@hotmail.ch

S. Clarke

e-mail: stephanie.clarke@chuv.ch

J. P. Thiran · A. Griffa

Signal Processing Laboratory, EPFL, Lausanne, Switzerland

R. Meuli · P. Hagmann

Service de Radiodiagnostic et Radiologie Interventionnelle, CHUV, Université de Lausanne, Lausanne, Switzerland human early-stage auditory cortical connectivity, as revealed by in vivo DSI tractography, has strong similarities with that of non-human primates. The modular architecture and hemispheric asymmetry in higher-order regions is compatible with segregated processing streams and lateralization of cognitive functions.

Keywords Auditory areas - Cortical connectivity . Human · Lateralization · Parallel and hierarchical processing

$\begin{array}{ll}\text { Abbreviations } \\ \text { AA } & \text { Anterior auditory area } \\ \text { ALA } & \text { Anterolateral auditory area } \\ \text { LA } & \text { Lateral auditory area } \\ \text { LP } & \text { Lateroposterior auditory area } \\ \text { MA } & \text { Medial auditory area } \\ \text { PA } & \text { Posterior auditory area } \\ \text { PAC } & \text { Primary auditory cortex } \\ \text { STA } & \text { Superior temporal auditory area }\end{array}$

\section{Introduction}

The human auditory cortex has been often described in terms of cortical regions found on the supratemporal plane, including Heschl's gyrus and large parts of the planum temporale and planum polare (von Economo and Horn 1930). Activation studies have confirmed the key role of the supratemporal plane and demonstrated on the temporoparieto-frontal convexity networks specialized in auditory functions such as sound recognition, sound localization or speech processing (Bushara et al. 1999; Maeder et al. 2001; 
Lewis et al. 2004; Hickok and Poeppel 2007; Belin 2006; Krumbholz et al. 2007).

In non-human species, the definition of the primary auditory area is based on a combination of criteria, including strong input from the medial geniculate body, cytoarchitecture, short-response latencies to auditory stimuli and an orderly tonotopic organization (Rose and Woolsey 1949). In non-human primates, the organization of cortico-thalamic connections and the presence of tonotopic maps lead to the identification of three primary and several non-primary auditory areas and, eventually, to the concept of auditory core, belt and parabelt (Pandya et al. 1994; Kaas and Hackett 2000). In humans, in view of the absence of precise data on cortico-thalamic connectivity, the functional subdivision of the supratemporal plane is based on architectonic studies and on tonotopic mapping. The primary auditory cortex (PAC), identified as koniocortex with prominent layer IV (area Te1), always covers portions of Heschl's gyrus, but there is no consistent and reliable association between its cytoarchitectonic boundaries and specific sulci (Rademacher et al. 2001). The role of Heschl's gyrus as landmark for PAC is, however, further confirmed by its high responsiveness to auditory stimuli (Viceic et al. 2009). Cytoarchitectonically, PAC comprises three subdivions which are arranged along the mediolateral axis of Heschl's gyrus (areas Te1.1, Te1.0 and Te1.2; Morosan et al. 2001). Tonotopic mapping revealed two tonotopic gradients on Heschl's gyrus, as well as additional tonotopic maps outside it (Da Costa et al. 2011; Langers 2014; for comprehensive review Saenz and Langers 2014). The surrounding, non-primary auditory cortex is cytoarchitectonically heterogeneous and has been subdivided into two (areas 42 and 22; Brodmann 1909, three Te 2.1, 2.2 and 3; Morosan et al. 2005, four TB, TA, TD and TG; von Economo and Horn 1930) or six areas (PaAi, PaAc/d, PaAe, PaAr, ProA, and Tpt; Galaburda and Sanides 1980). Functionally related stains of cytochrome oxidase, acetylcholinesterase and NADPH-diaphorase activity as well as immunohistochemical revelation of parvalbumine revealed a consistent pattern of seven non-primary areas, whose cortical surface varied between 1.1 and $3.1 \mathrm{~cm}^{2}$; these areas are referred to as posterior auditory area (PA), lateral auditory area (LA), lateroposterior auditory area (LP), anterolateral auditory area (ALA), anterior auditory area (AA), medial auditory area (MA) and superior temporal auditory area (STA); Fig. 1a; (Rivier and Clarke 1997; Wallace et al. 2002; Chiry et al. 2003). Distinctive features of the staining patterns, such as prominence of a midcortical band in cytochrome oxidase staining or the proportion of fiber vs. somatic acetylcholinesterase staining were compatible with a hierarchical three-tier organization of (i) PAC; (ii) 6 intermediate level areas (PA, LP, LA, ALA, AA, MA); and (iii) at least one higher level area (STA).
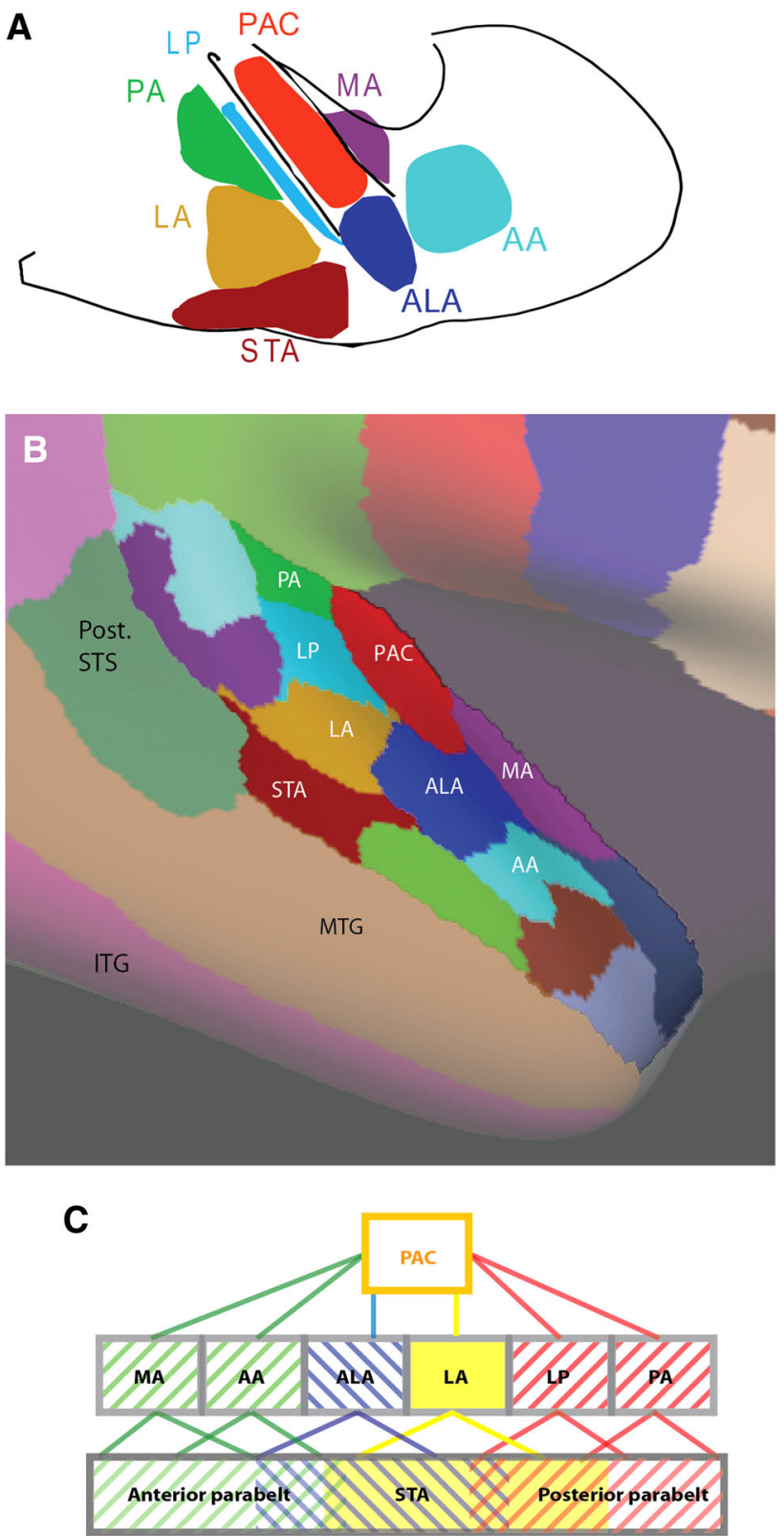

Fig. 1 Architectonically identified early-stage auditory areas on a upper view of the right supratemporal plane (a); adapted from Rivier and Clarke 1997; Wallace et al. 2002); as identified in the present study on the right inflated temporal lobe (b); and in a schematic representation of their connectivity (c). For area designations, see list of abbreviations. ITG inferior temporal gyrus, $M T G$ middle temporal gyrus, Post. STS posterior part of superior temporal sulcus

Currently, very little is known about the cortico-cortical connectivity of the human auditory cortex. Two postmortem studies using DiI or DiA described intrinsic connections within primary and non-primary early-stage auditory areas (Tardif and Clarke 2001) and within Brodmann area 22 (Galuske et al. 2000). Two in vivo studies, using DTI, described functionally relevant fiber tracts originating in Heschl's gyrus: interconnections within 
Heschl's gyrus between high- and low-frequency representations (Upadhyay et al. 2007) and on the supratemporal plane and superior temporal gyrus between foci of effective connectivity as identified during listening to short sentences (Upadhyay et al. 2008). The pattern of connectivity between and beyond human early-stage auditory areas and its relationship to putative processing streams is, however, largely unknown.

In non-human primates, cortico-cortical connections codefine hierarchical levels of processing within the auditory cortex. Tracing studies have demonstrated a cascade-like connectivity pattern between the core which contains the primary areas $\mathrm{AI}$ and $\mathrm{R}$, the belt which flanks the core laterally and medially and which contains areas AL, ML, CL, CM and RM, and the parabelt which is lateral to the (lateral) belt and is subdivided into rostral and caudal parts (Kaas et al. 1999). AI has strong reciprocal connections with $\mathrm{R}$ as well as with the belt, but not with the parabelt areas; the belt areas have strong reciprocal connections with each other, with the core and with the parabelt; and the parabelt has strong connections with the belt, but not the core (e.g., Morel et al. 1993; de la Mothe et al. 2006; Hackett et al. 1998). The parabelt and the adjacent superior temporal gyrus have long intrahemispheric connections with prefrontal cortex (e.g., Romanski et al. 1999; Hackett et al. 1999) and they receive in their caudal parts somatosensory input (Smiley et al. 2007; Hackett et al. 2007).

We have carried out a systematic investigation of human auditory cortex connectivity using in vivo DSI tractography combining landmark-based registration, automatic cortical parcellation and whole-brain structural connection matrices (Cammoun et al. 2012; Hagmann et al. 2008). Our working hypothesis stipulated that the pattern of connectivity would be related on the supratemporal plane to the architectonically identified early-stage auditory areas ( $\mathrm{Ri}$ vier and Clarke 1997; Wallace et al. 2002) and beyond it to regions of similar size. To test this hypothesis, we subdivided each hemisphere into 250 regions of interest (ROIs); (Cammoun et al. 2012). On the supratemporal plane, we determined ROIs whose Talairach coordinates corresponded to the eight architectonically defined early-stage auditory areas (Rivier and Clarke 1997; Wallace et al. 2002) and we then analyzed the connectivity pattern of each early-stage auditory area and of each ROI within the temporo-parieto-frontal cortex.

\section{Materials and methods}

Twenty right-handed healthy men aged from 22 to 35 years participated in this study. None had a history of psychiatric or neurological illness and all reported normal hearing. The experiments were approved by the Ethics Committee of the Faculty of Biology and Medicine of University of Lausanne; all subjects provided written, informed consent to participate in this study.

MRI acquisition and connectome construction

The imaging protocol was performed using a Siemens TimTrio 3T scanner (with a 32-channel receive head matrix coil). We used a diffusion-weighted single-shot EPI sequence with a TR of $6,000 \mathrm{~ms}$ and a TE of $138 \mathrm{~ms}$, and a maximal $b$ value of $8,000 \mathrm{~s} / \mathrm{mm}^{2} .256$ diffusion-weighted images were acquired with varying $b$ value and diffusionsensitizing gradient direction covering a hemi-ball in a Cartesian 3D grid in $q$ space. The reconstruction of the data was done according to the standard diffusion spectrum imaging (DSI) protocol (Wedeen et al. 2005). In addition, a high-resolution T1-weighted (MP-RAGE) MRI was acquired in a matrix of $256 \times 256 \times 128$ voxels of isotropic $1 \mathrm{~mm}$ resolution. The processing pipeline producing the connection matrix comprised two pathways (Cammoun et al. 2012): (i) extraction of the cortical surface from a high resolution $\mathrm{T} 1$-weighted image and its subdivision into 250 ROIs per hemisphere; and (ii) whole brain tractography with the reconstruction of virtual fibers throughout the brain. The combination of these two procedures allowed the construction of connection matrices by computing the number of virtual generated fibers connecting each pairs of ROIs.

\section{Cortical subdivision}

The cortical surface was extracted from a high resolution T1-weighted image (Fischl et al. 2004). The technique available in the Freesurfer freeware (http://surfer.nmr.mgh. harvard.edu/) assigned a neuroanatomical label to each location on a cortical surface model based on probabilistic information estimated from a manually labeled training set (Desikan et al. 2006), identifying gyral-based "parcels". Each parcel was subdivided into smaller cortical ROIs with a cortical surface area of $3.5 \pm 0.4 \mathrm{~cm}^{2}$ and $1 \mathrm{~mm}$ thickness; the entire cortex of one hemisphere comprised 250 ROIs (Cammoun et al. 2012).

On the supratemporal plane, we have identified eight ROIs whose Talairach coordinates corresponded to those of the previously described early-stage auditory areas PAC, AA, ALA, LA, PA, MA and STA; these areas have been defined in histological sections on the basis of their cytoand myeloarchitecture as well as their distinct staining patterns of cytochrome oxidase, acetylcholinesterase, NADPH-diaphorase and parvalbumin immunohistochemistry and their Talairach coordinates have been published (Rivier and Clarke 1997; Wallace et al. 2002; Chiry et al. 
2003). These eight ROIs are referred to in this paper as areas PAC, AA, ALA, LA, PA, MA and STA. All other ROIs are described in terms of their anatomical location (e.g., ROIs on the posterior part of the middle temporal gyrus). The areas and ROIs, which were investigated here, were grouped into 20 anatomical regions: transverse temporal gyrus; anterior and posterior parts of the superior temporal gyrus; anterior and posterior parts of the middle temporal gyrus; inferior temporal gyrus; superior temporal sulcus; temporal pole; entorhinal and parahippocampal cortices; supramarginal and angular gyri; anterior and posterior parts of middle frontal gyrus; partes orbitalis, triangularis and opercularis of inferior frontal gyrus; lateral and medial parts of orbitofrontal cortex; and frontal pole. Within a given anatomical region, all ROIs were included in our analysis and not only those explicitly known for their involvement in auditory processing: e.g., all ROIs of the temporal pole were included and not only the dorsal ones (Ding et al. 2009). When represented in a matrix, the order of the anatomical regions followed as much as possible anatomical proximity, starting at the temporal and finishing at the frontal pole.

\section{White matter tractography}

Tractography is a post-processing method using the diffusion-weighted images and based on the molecular displacement of water molecules under strong magnetic fields. The Diffusion MRI scans produce a signal corresponding to the Fourier transform of the diffusion probability density function (PDF) at every point in the brain in accordance to the used resolution. Using this relationship between MR signal and PDF, the local diffusion PDF could be recovered for every imaged brain location (Hagmann et al. 2006). The tractography algorithm results are three-dimensional curves of maximal diffusion coherence. These curves, called virtual fibers, are the estimates of the real white matter axonal bundle trajectories. We used a tractography algorithm specifically designed for DSI data to create a set of such fibers for the whole brain (Hagmann et al. 2007; Wedeen et al. 2008). Although DSI data were shown to provide better appreciation of fiber trajectories at bundle crossings than DTI data (Gigandet 2009), the crossing fiber problem is likely to persist. This is a well-known problem for interhemispheric fibers, which pass through the body of the corpus callosum and cross on their way the corona radiata. It is a lesser problem for intrahemispheric cortico-cortical pathways, which tend to be reliably reconstructed (Schmahmann et al. 2007). For this reason we focused our study on intrahemispheric connections.
Construction of the connection matrix

Considering the cortical parcellation and the white matter tractography described in the previous paragraphs, the fiber bundle $B(i, j)$ connecting each pair of ROI $i$ and $j$ could be identified. The value of the connection matrix cell $M(i, j)$ was the connection density e.g. the number of virtual fibers connecting this pair of ROIs, and defined as follows: $M(i, j)=\sum_{f \in B(i, j)} 1 / l(f)$, where $l(f)$ is the length of fiber $f$ along its trajectory. The correction term $l(f)$ in the denominator is needed to eliminate the linear bias towards longer fibers introduced by the tractography algorithm (Hagmann et al. 2007), which uses each voxel in the white matter mask as a seed point. As the number of seed points in the tractography algorithm is corresponding to the white matter volume an additional normalization of the matrix by this volume is required for a group study. This additional normalization would correct the bias due to the fact that a small brain has less fiber than a big one in general.

In order to avoid false positives, i.e., reporting unlikely connections, the identified fibers were filtered on the basis of their mean fractional anisotropy (FA) along their trajectory. A global histogram of all subjects helped to identify empirically a subpopulation with small FA $(<0.1$ for FA normalized between 0 and 1), which was considered diffusion noise and served to define the threshold. Auditory connectivity was then analyzed in two ways. In a first approach, the considered measurement in the matrix was the number of fibers running between each pair of ROI after the filtering based on FA. In a second approach, connectivity patterns were assessed in terms of connecting fibers. In each individual subject, the number of fibers running between each two ROIs were calculated after the filtering by FA and binarized ( 0 if less than FA threshold, 1 otherwise) to inform simply each time if there is a connection between the pair of ROIs or not for individual subject. The population connectivity matrix was calculated as the mean of individual matrices. Connectivity of individual early-stage auditory areas and other ROIs were fixed in the template, and the intensity of connectivity from each of these ROIs to the rest of the matrix was calculated and plotted on an average cortex to visualize the topography of the auditory connectivity network.

Graph theory (Sporns 2011) was used to analyze the auditory subnetwork on the supratemporal plane and parts of the superior temporal gyrus. This submatrix comprised the primary auditory cortex, the six belt areas and eight parabelt areas (as shown in Fig. 2). The 15 ROIs were considered nodes, whereas edges consisted of connections between pairs of ROIs. The auditory areas were represented by binary undirected graphs. The connectivity pattern within these subregions was compared with that of 
random graph models, which were the results of 100 randomizations of each subject's brain, which shared the size, binary degree distribution of the original network (Maslov and Sneppen 2002). Three characteristics were analyzed. First, the cascade-like pattern was evaluated by comparing connections travelling from PAC to parabelt (PB) via belt (B) to direct connections from PAC to parabelt. To test this hypothesis, we defined a score describing the number of connections necessary to connect ROIs of PAC directly to ROIs of parabelt, W_PAC_PB, and the $z$-score for connections traveling via belt ROIs W_PAC_B_PB as follow: modules per hemisphere. The mean module structure within our population of 20 normal subjects was then determined as follows. For either hemisphere of each subject, we identified modules which dominated one of the following five regions: (i) the supratemporal plane (comprising transverse gyrus and the anterior and posterior STG); (ii) the temporal convexity (anterior and posterior parts of MTG and ITG); (iii) inferior parietal convexity (angular gyrus, supramarginal gyrus, inferior parietal gyrus); (iv) upper part of the frontal convexity (superior and middle frontal gyri); and (v) lower part of the frontal convexity (inferior frontal gyrus, frontal pole).

$$
Z_{P A C-B-P B}=\frac{\left(W_{P A C-B-P B}-\overline{W_{P A C-B}-P B}\right)}{s t d\left(\overline{\left.W_{P A C-B-P B}\right)}\right)} ; Z_{P A C-P B}=\frac{\left(W_{P A C-P B}-\overline{W_{P A C-P B}}\right)}{s t d\left(\overline{W_{P A C-P B}}\right)}
$$

where $\widetilde{W_{P A C_{-} P B}}$ and $W_{P A C-B} \widetilde{ }$, respectively, are the scores describing the number of connections necessary to connect PAC to parabelt directly and the PAC to parabelt via belt areas in the generated random networks. Second, the degree of integration was assessed by global efficiency score within the auditory sub-network (i.e., the average inverse shortest path length in the network). The global efficiency is used as a measure of the overall capacity for parallel information transfer and integrated processing. It is a topological measure of the reciprocal or inverse path length between nodes (Bullmore and Sporns 2012). Third, the degree of segregation was assessed by clustering coefficient. This network measurement captures the cliquishness of a local neighborhood, based on the number of triangular connections between groups of three nodes (Bullmore and Sporns 2012), (i.e., the fraction of nodes whose neighbors are also neighbors of each other (Watts and Strogatz 1998). Segregated process should benefit from highly clustered connections, whereas integrated processes should benefit from high global efficiency of information transfer across the network as a whole (Bullmore and Sporns 2012). For each subject, a set of 100 random graphs with the same size and degree distribution were generated using the brain connectivity toolbox (BCT), the clustering coefficient and the global efficiency of subjects were compared with a paired $t$ test to their mean random network homologous attributes.

The optimal community structure, a subdivision of a network into non-overlapping groups of nodes in a way that maximizes the number of within group edges and minimizes the number of between group edges, was determined using the spectral community detection algorithm described in (Newman 2006). The optimal community structure was determined in each single subject, based on the mean density of connections, which led to the identification of 4-8
The mean distribution of modules $i$ to $\mathrm{v}$ was then determined in each hemisphere.

The trajectories of fibers originating/terminating from individual ROIs were visualized with Trackvis toolkit (http://trackvis.org/) on the basis of virtual fibers generated for the construction of the connection matrix; a 3D dilation was applied to a given ROI, the structuring element being a sphere of radius 1).

Hemispheric asymmetry in cortico-cortical connectivity

Hemispheric asymmetry in cortico-cortical connectivity was assessed by comparing the number of fibers which interconnected, in each hemisphere, 20 anatomical regions [defined in terms of gyral-based "parcels"; (Desikan et al. 2006)]. To preserve anatomical proximity and putative functional specialization within the matrix, two of the original parcels, the superior and middle temporal gyri, were subdivided into anterior and posterior parts in respect to Heschl's gyrus. The number of interconnecting fibers was normalized per subject between 0 and 1 . The $20 \times 20$ connection matrices of the right and the left hemisphere were compared using a paired $t$ test $(p<0.05)$ and a false discovery rate was applied as correction for multiple testing (Hochberg and Benjamini 1990).

\section{Results}

Connectivity within the supratemporal plane and the superior temporal gyrus

Analysis of interareal connections on the supratemporal plane revealed a cascade originating in PAC and 

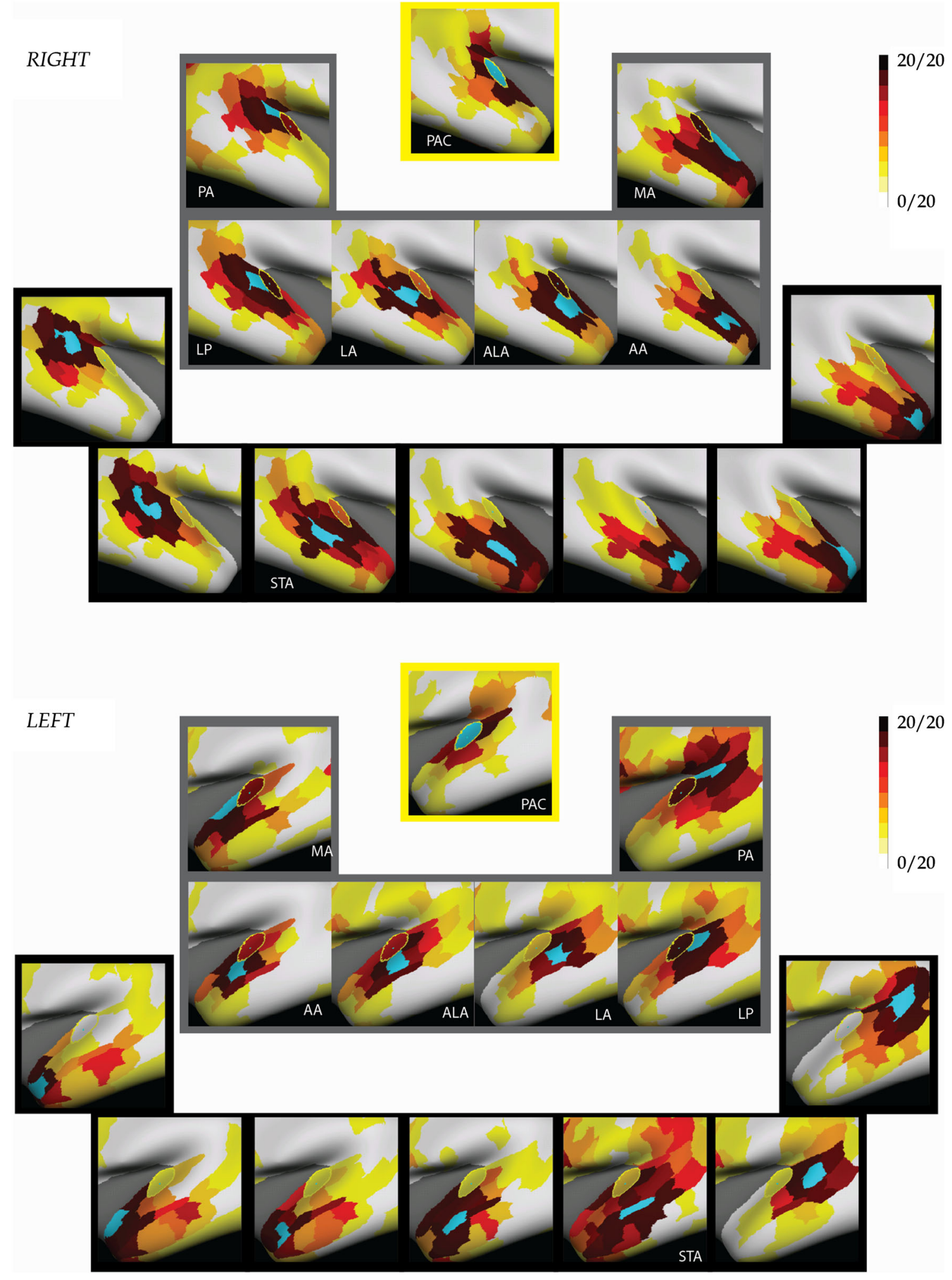
4Fig. 2 Connections of the primary auditory cortex $(P A C)$, the belt areas (insets outlined in gray MA, AA, ALA, LA, LP, PA), and the parabelt (insets outlined in black STA and other, unnamed ROIs) in the right and left hemispheres (top and bottom panels respectively). The area whose connectivity is represented in a given inset is in cyan; PAC is outlined in yellow; brown to yellow represents strength of the connectivity $($ black $=$ present in all 20 cases; yellow $=$ present in 1 of the 20 cases). The insets are arranged according to the position of the represented area (in cyan); cyan areas rostral to PAC, such as MA or AA, are placed rostrally to the central panel (with PAC), i.e., on the right side in the top panel and on the left side in the bottom panel. Note that PAC is strongly interconnected with the adjacent belt areas (PA, MA, LA, LP ALA, and AA). The belt areas are strongly interconnected with (i) PAC; (ii) the adjacent belt areas; and (iii) the nearby parabelt ROIs. The latter are only rarely interconnected with PAC; their strongest connections were with the neighboring belt and ROIs

propagating throughout the early-stage areas toward the superior temporal gyrus. PAC was strongly interconnected with the immediately surrounding non-primary areas MA, AA, ALA, LA, LP and PA, whereas only weak connections were present to cortical regions beyond these non-primary areas, such as STA and the proximal parts of the planum temporale (Fig. 2).

The connectivity patterns of non-primary areas depended on their position in respect to PAC. Areas MA, AA, ALA, LA, LP and PA, which are immediately adjacent to PAC (Fig. 2; insets outlined in gray), are very likely homologs of the belt areas described in non-human primates (Kaas et al. 1999). They had strong interconnections with (i) PAC; (ii) the adjacent belt areas; and (iii) the neighboring ROIs lying more laterally on the superior temporal gyrus. Thus, ALA had strong connections to PAC, LA, AA and MA as well as to the ROIs lying further away on the planum polare and on the middle part of the superior temporal gyrus. The rostrally lying areas ALA, AA and MA had strong interconnections within the planum polare, spreading up to the temporal pole. The caudally lying LP and PA had the strongest connections within the planum temporale and the posterior part of the superior temporal gyrus.

Adjacent to the putative belt areas lay a crescent of ROIs (Fig. 2, insets outlined in black), which is likely to be the putative homolog of non-human parabelt areas (Kaas et al. 1999). These areas were characterized by no or only scarce connections with PAC and by strong interconnections with (i) the neighboring belt areas; (ii) the adjacent ROIs within the parabelt; and (iii) the neighboring ROIs lying more laterally on the superior temporal gyrus. An individual ROI within the parabelt tended to be interconnected with more areas or ROIs than was PAC or an individual belt area. Rostrally lying ROIs tended to be interconnected with the planum polare and with ROIs on the anterior part of the temporal convexity and the temporal pole, caudally lying
ROIs with the planum temporale and with ROIs on the posterior part of the temporal convexity and the parietal operculum. Several ROIs had strong connections to prefrontal regions.

Inspection of the connection patterns of individual areas and ROIs suggested that PAC is connected to the parabelt areas mainly via belt areas. We have tested this hypothesis by assimilating the 15 areas of the PAC-belt-parabelt region to a network. In either hemisphere of each subject, the number of direct connections between PAC and the parabelt, and the number of connections traveling via the belt were compared to those in networks generated by 100 randomizations of the same subject's brain. The high $z$-score values for $Z_{\text {PAC-B-PB }}$ and low for $Z_{\text {PAC-PB }}$ (Table 1) confirmed the connectivity architecture hypothesis. The number of direct connections between PAC and parabelt was similar to that predicted from random connectivity, whereas the number of connections going from PAC to the parabelt via the belt exceeded by large that predicted by a random connectivity organization, suggesting a cascadelike connectivity.

The connectivity patterns illustrated in Fig. 2 suggested that PAC, the belt and the parabelt be the origin of separate processing streams. We have analyzed the pattern of connectivity within these subregions with the Graph theory approach; our results supported the hypothesis that the auditory network constituted by PAC, the belt and the parabelt, has a strong degree of segregation. Clustering coefficient was significantly larger in subjects than in randomized networks within the right $\left(p=4.9 \times 10^{-16}\right)$ and the left hemisphere $\left(p=1.2 \times 10^{-21}\right)$, suggesting a high degree of segregation according to the postulate that segregated processes benefit from highly clustered connections (Bullmore and Sporns 2012). The integration of the PAC-belt-parabelt network was assessed by the global efficiency measurement, which was significantly larger in subjects than in randomized networks within the right $\left(p=1.3 \times 10^{-19}\right)$ and the left hemisphere $\left(p=4 \times 10^{-23}\right)$ indicating a high degree of integration. We also measured hierarchy using global reaching centrality (Mones et al. 2012), which was significantly higher in the right $\left(p=4.6 \times 10^{-6}\right)$ and left hemispheres $\left(p=2.4 \times 10^{-5}\right)$ in subjects than in generated random networks (Table 2).

\section{Connectivity of the tempo-parietal convexity}

Individual ROIs on the temporal convexity tended to have strong and relatively wide-spread connections to the neighboring ROIs, but not to PAC and only rarely to belt areas (Fig. 3). In addition to the local connectivity some, but not all ROIs had distal connections to the posterior part of the inferior frontal gyrus. Individual ROIs on the inferior 


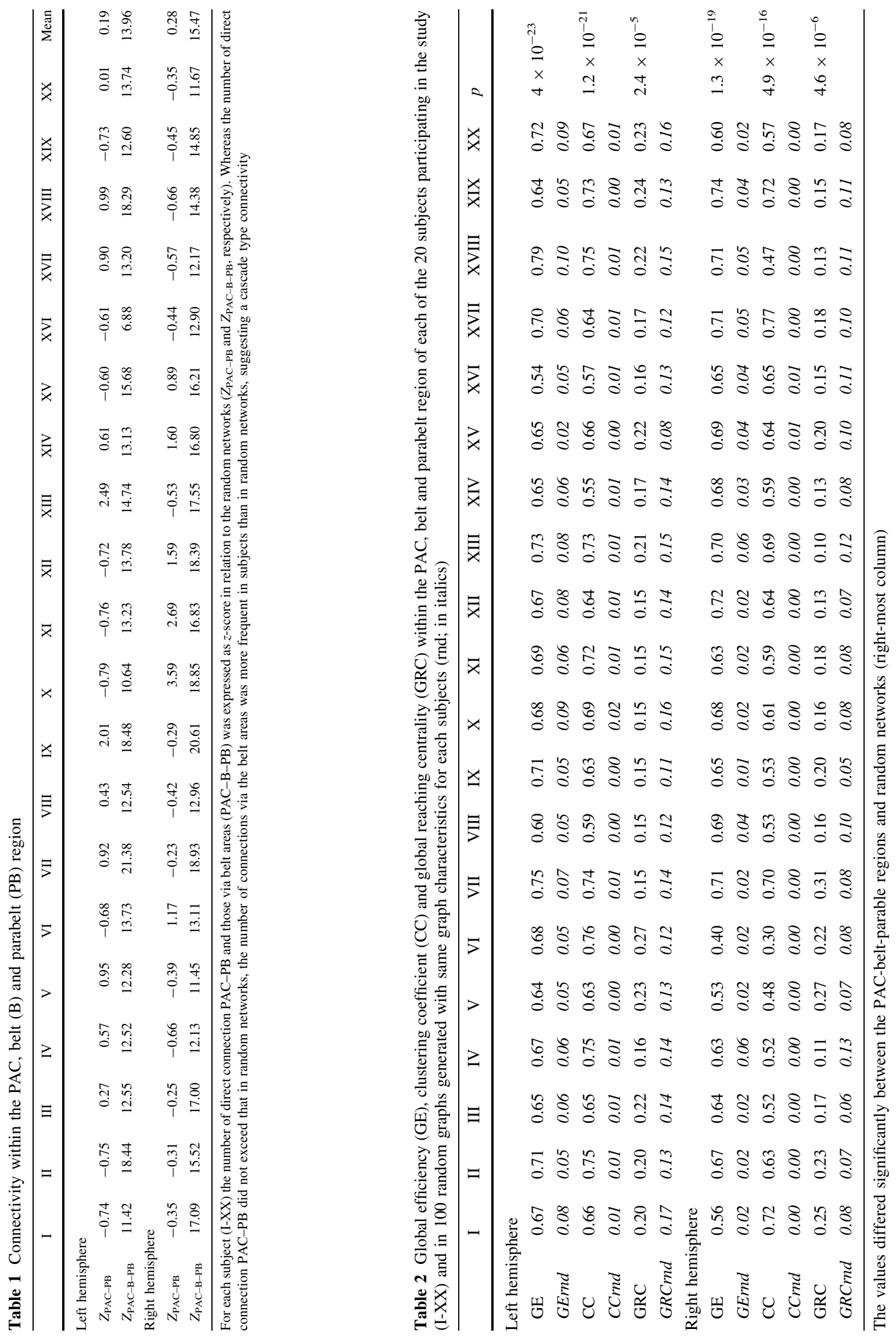


parietal lobule tended to have strong local connections to the neighboring ROIs and to the caudal part of the prefrontal convexity. These connectivity patterns are illustrated for ROIs on the anterior and posterior parts of the middle temporal gyrus and on the superior and inferior parts of the supramarginal gyrus of the left and the right hemisphere (Fig. 3, top panel). The fibers which constituted these connections were part of the perisylvian fiber system (Fig. 3, bottom left panel), including the arcuate and superior longitudinal fascicule (Catani et al. 2002, 2005).

\section{Modularity of auditory connections}

The clustered connectivity of ROIs on the tempo-parietal convexity suggests a putative organization into processing streams. We have tested this hypothesis by determining the optimal community structure, which corresponds to the subdivision of the network into non-overlapping groups of nodes (i.e. ROIs) by maximazing the number of within group edges (i.e. connections) and minimizing the number of between group edges. This calculation was preformed separately for either hemisphere of each subject. Distinct modules were consistently found in five regions, which are known to be involved in auditory processing: (i) the earlystage auditory areas on the supratemporal plane; (ii) the temporal convexity; (iii) the inferior parietal convexity; (iv) the upper part of the frontal convexity; and (v) the lower part of the frontal convexity. For each of the five regions, we identified in each individual hemisphere the module which included most of the ROIs. The mean distribution of these modules, as calculated across all subjects, showed a similar configuration in the two hemispheres (Fig. 3). The module centered on the supratemporal plane (in blue) covered mainly Heschl's gyrus and the planum temporale; the module centered on the temporal convexity (in green) included also the planum polare and the temporal pole as well as small parts of the inferior frontal cortex; the module centered on the parietal convexity (in red) spread also onto the posterior parts of the middle and inferior temporal gyri; the module centered on the upper part of the frontal convexity (in mauve) included most of the middle frontal gyrus, and the module centered on the lower part of the frontal convexity (in cyan) covered most of the inferior frontal gyrus and spread towards the orbito- and mediofrontal cortex.

Hemispheric differences of auditory cortico-cortical connections

Hemispheric differences in cortico-cortical connectivity were assessed by comparing, in each hemisphere, fiber density between 20 anatomical regions which were defined in terms of gyral-based "parcels" (Desikan et al. 2006); Fig. 4. A leftward advantage was present for several ventro-dorsal connections, mainly those involving the posterior part of the superior and middle temporal gyri and the angular and supramarginal gyri. A rightward advantage was present for several antero-posterior connections, involving the anterior part of the middle temporal gyrus.

\section{Discussion}

Investigating cortical connections in man

Currently, there are two approaches to the investigation of neural connectivity of the human cerebral cortex. Postmortem studies, using different anterograde or retrograde tracers, visualized specific axonal pathways, often individual axons, as well their neurons of origin and/or axonal terminal fields. This approach was successfully applied to the human auditory cortex for unraveling the finer organization of cortical connectivity, but not for assessing the complex pattern of temporo-parieto-prefrontal connections. Anterograde and retrograde tracing with DiI or BDA, limited to short connections (below 10-15 mm), has been used for intracortical connections of the auditory and associated cortices (Tardif and Clarke 2001; Tardif et al. 2008; Galuske et al. 2000). Long heterotopic interhemispheric connections have been traced from the right inferotemporal cortex to Wernicke's and Broca's areas using the Nauta method for anterogradely degenerating axons (Di Virgilio and Clarke 1997). Although the Nauta method visualizes very reliably monosynaptic pathways (Di Virgilio et al. 1999; Clarke 1994; Clarke and Miklossy 1990; Di Virgilio and Clarke 1997; Clarke et al. 1999; Wiesendanger et al. 2004), its application depends on the location and extent of the lesion which causes anterograde axonal degeneration. Unfortunately, lesions occurring within the perisylvian region are often large, multiple and/or encroaching on the numerous subcortical tracts which are found in the vicinity of the auditory cortex (Bellmann et al. 2001; Thiran and Clarke 2003), which limits the use of the Nauta method. Myelin degeneration studies suffer from the same drawback and were further limited by their relatively low sensitivity (Miklossy et al. 1991).

A second promising approach has been developed on the basis of DTI and DSI tracing, investigating most often whole brain connectivity. Both DTI and DSI have been validated by comparisons with histological tracing techniques. Good anatomical correspondence has been found between the location of major fiber tracts of the human brain as identified by DTI tractography and by microscopy in myelin-stained histological sections (Thiebaut de Schotten et al. 2011; Burgel et al. 2006). High level 


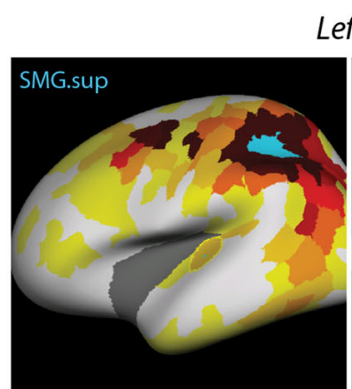

\section{eft}
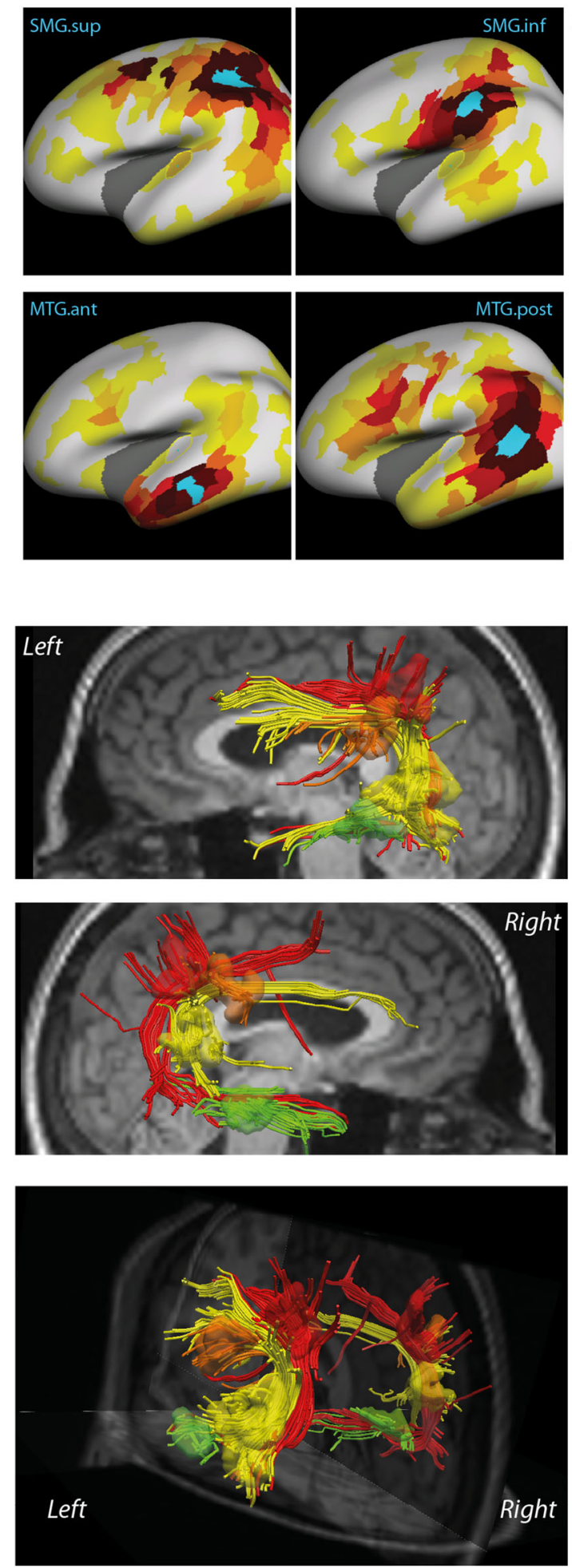

\section{Right}
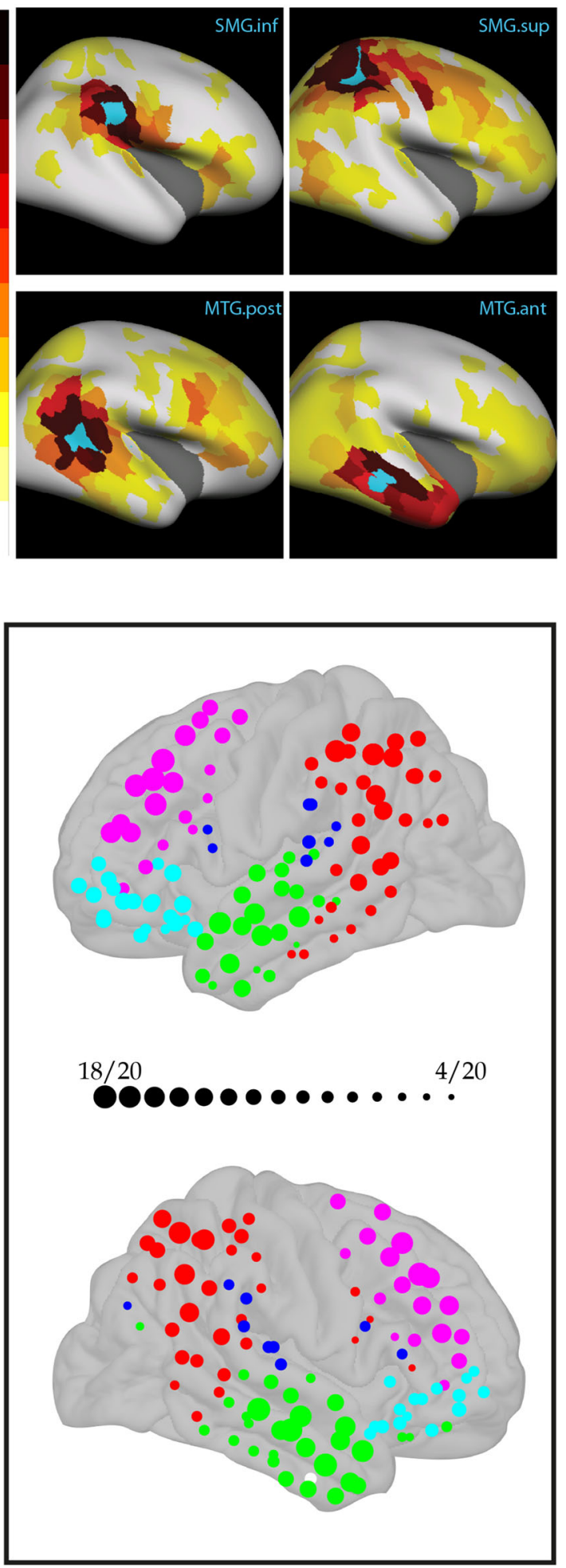
4Fig. 3 Cortico-cortical connectivity within the left and right hemispheres (in left and right columns, respectively). Top panel connectivity of ROIs on the superior and inferior parts of the supramarginal gyrus (sup SMG, inf SMG) and the anterior and posterior parts of the middle temporal gyrus (ant MTG, post MTG). Same color conventions as in Fig. 2. Bottom left panel visualization of cortico-cortical fibers originating in ROIs on the superior and inferior parts of the supramarginal gyrus (red and orange, respectively) and on the anterior and posterior parts of the middle temporal gyrus (green and yellow, respectively; same ROIs as in top panel), in a typical subject. Bottom right panel modules of cortico-cortical connectivity as determined by optimal community structure. Colors denote mean distribution of modules defined in individual hemispheres as centered on (i) the supratemporal plane (blue); (ii) the temporal convexity (green); (iii) the parietal convexity (red); (iv) the upper part of frontal convexity (mauve); and (v) the lower part of frontal convexity (cyan). Circles represent ROIs; the size of the circle is proportional to the number of subjects in whom the ROI participated in the corresponding module evidence is available for the reliability of DSI. The highly sensitive autoradiographic tracing technique, generally considered as gold standard for tract tracing, leads to the identification of fiber tracts in the macaque brain (Schmahmann and Pandya 2006); these same fiber tracts have been also identified in the living macaque by means of DSI tracing (Schmahmann et al. 2007).

Cortico-cortical connectivity derived from human DSI data was shown to have characteristics of complex networks, such as small-world topology, connector and provincial hubs, and modularity (Bullmore and Sporns 2009). Within this analytical framework, auditory cortex was described as part of a temporal module, which was defined by the strong connectivity between the supratemporal plane and the temporal convexity; provincial hubs were identified
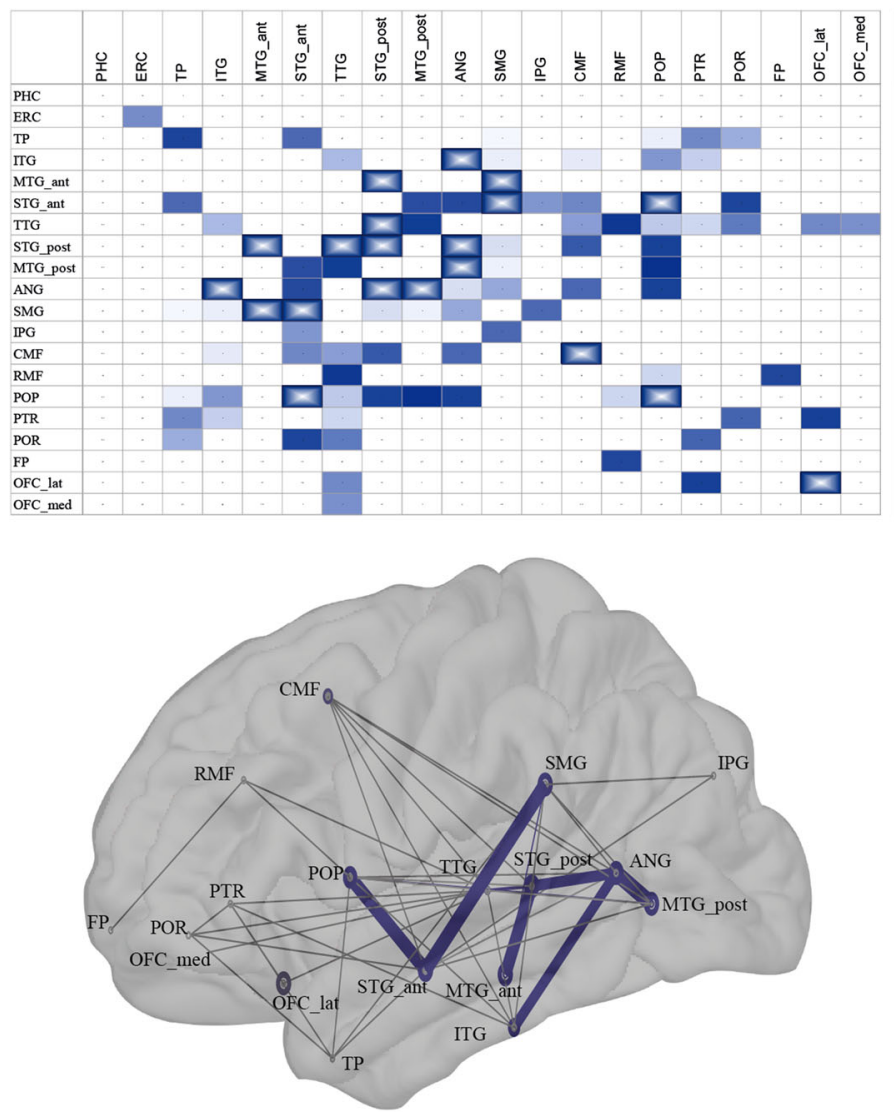

Fig. 4 Hemispheric asymmetry of cortico-cortical connections. The matrices of 20 anatomical regions defined by gyral patterns highlight in hues of blue and red interconnections, which were significantly denser in the left and right hemisphere, respectively $(p<0.05)$. White asterisks indicate connection asymmetries, which remained significant after false discovery rate (FDR) was applied as correction for multiple testing. The brain insets represent schematically connections which are significantly denser in the corresponding hemisphere (thick lines significant after FDR correction). Abbreviations denote anatomical regions: $A N G$ angular gyrus, ant anterior part, $C M F$ caudal
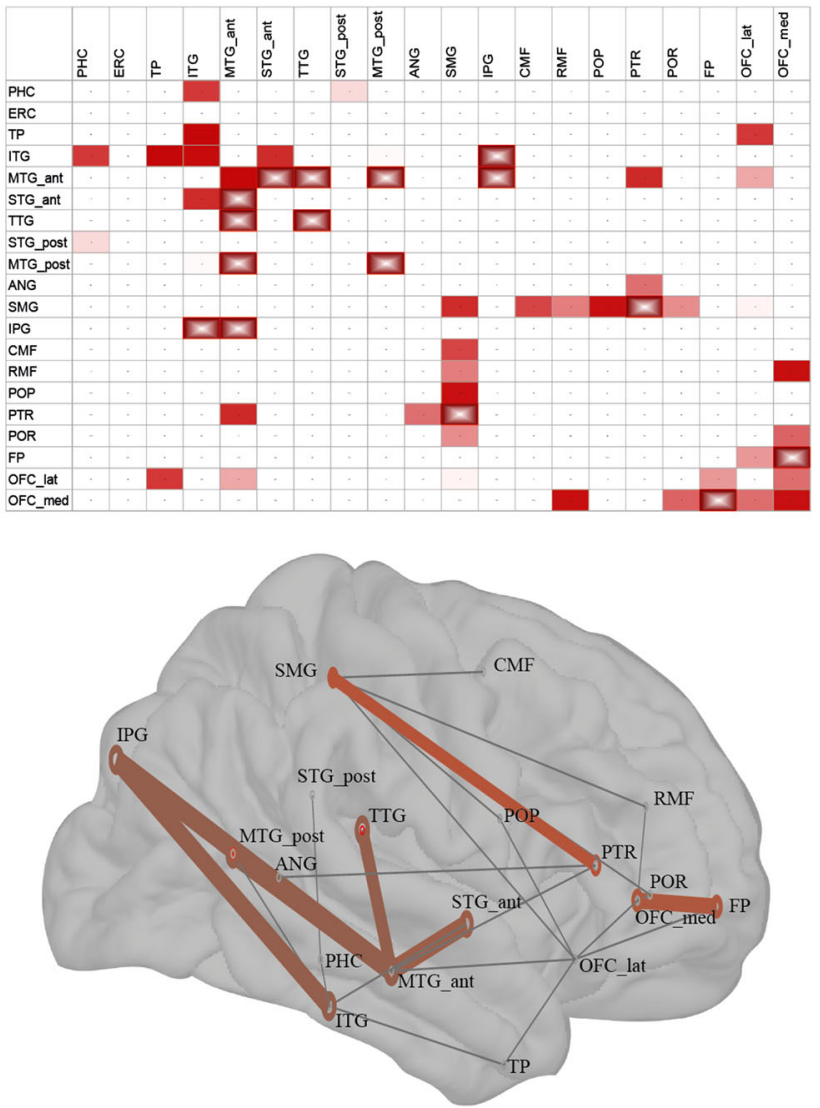

medio-frontal cortex, $E R C$ entorhinal cortex, $F P$ frontal pole, $I F G$ inferior frontal gyrus, IPG inferior parietal gyrus, ITG inferior temporal gyrus, lat lateral part, med medial part, $M F G$ middle frontal gyrus, $M T G$ middle temporal gyrus, $O F C$ orbitofrontal cortex, $P H C$ parahippocampal cortex, $P O P$ pars opercularis (of IFG), $P O R$ pars orbitalis (of IFG), post posterior part, PTR pars triangularis (of IFG), $R M F$ rostral medio-frontal cortex, $S M G$ supramarginal gyrus, $S T G$ superior temporal gyrus, STS superior temporal sulcus, TP temporal pole, $T T G$ transverse temporal gyrus 
in this module on the superior and middle temporal gyri, the superior temporal sulcus and the supramarginal gyrus (Hagmann et al. 2008). Another DTI study with a large number of subjects identified nine major hubs as well as primary and association nodes, but failed to characterize auditory-related networks (Gong et al. 2009).

We have combined here the two approaches and tried to unravel the finer organization of early-stage auditory areas, by linking DSI-derived connectivity to histologically identified auditory areas on the supratemporal plane and by comparing patterns of local and distal connectivity within different parts of the temporo-parieto-frontal cortex. Our results revealed a pattern of auditory cortical connectivity, which is compatible with parallel and hierarchical processing.

\section{Connectivity of human early-stage auditory areas}

The present data strongly support parallel and hierarchical organization within the early-stage auditory areas with three distinct tiers which share similarities with the core, belt and parabelt regions identified in non-human primates (Kaas et al. 1999). Our results have shown a two-step cascade of connections: (i) PAC to areas MA, AA, ALA, LA, LP and PA, which can be considered to be putative homologs of non-human primate belt areas; and (ii) belt areas to a crescent of immediately adjacent ROIs, including area STA, which can be considered to be putative homo$\log$ of non-human parabelt areas (Fig. 1c). As in nonhuman primates, there are strong interconnections between core and belt and between belt and parabelt, but not between core and parabelt. The six belt areas tend to have topographically distinct patterns of connectivity with the parabelt and beyond it with the temporo-parietal convexity.

Supporting evidence for a hierarchical relationship between PAC and the belt and parabelt areas and their interconnections comes from preoperative recordings. A cascade of latencies to auditory stimuli was reported between PAC (latencies of 30-50 ms) and the anterolateral part of Heschl's gyrus, which corresponds very likely to ALA (Liegeois-Chauvel et al. 1994). Electrical stimulations in PAC yielded 6-8 ms later neural responses in the anterolateral part of Heschl's gyrus and posterolateral to it, corresponding very likely to ALA and PA, respectively (Liegeois-Chauvel et al. 1991). Shorter latencies, 2-3 ms, were reported in a similar paradigm in the upper part of the superior temporal gyrus, corresponding very likely to STA (Howard et al. 2000). Waveform analysis from multisite recordings upon biphasic electrical pulses to PAC strongly suggests a serial processing from PAC to LA and then to STA (Brugge et al. 2003, 2008). Functional connections from PAC to belt and parabelt areas were revealed also by directed coherence analysis of auditory-evoked potentials in response to amplitude-modulated sounds; these results are compatible with serial and parallel processing streams at the level of early stage auditory areas (Gueguin et al. 2007; Gourevitch et al. 2008).

Several early-stage areas are likely to be tonotopically organized, and their organization may underlie inter-areal connectivity patterns. Presently the orientation of tonotopic gradients differs between studies, and the correspondence with architectonically identified areas still needs to be established (Schonwiesner et al. 2002; Talavage et al. 2004; Humphries et al. 2010). A recent 7 T fMRI study demonstrated two tonotopic gradients on the central part of Heschl's gyrus, suggesting the existence of two core areas within the PAC, which may be homologs of macaque primary areas AI and R (Da Costa et al. 2011). The connectivity of PAC, reported here, thus reflects the connections of two distinct core areas. To disentangle the respective connection patterns of $\mathrm{AI}$ and $\mathrm{R}$, combined tonotopical mapping and tract tracing needs to be carried out with 7 T MRI. The existence of additional core areas is suggested by a recent tonotopic investigation of the human auditory cortex using volumetric and surface-based cortical alignments (Langers 2014); judging by their position with respect to Heschl's gyrus, these areas may correspond to LP, ALA and MA. Our study shows a strong connectivity between PAC and these three areas in the right, but not the left hemisphere. The connectivity between one of these putative core areas and PAC appears to be tonotopically organized; a DTI study demonstrated a tonotopic organization of connections between PAC and the more lateral part of Heschl's gyrus, corresponding very likely to ALA (Upadhyay et al. 2007); intra-areal connectivity within PAC between representations of different frequencies was found to be stronger than inter-areal connectivity between tonotopically corresponding parts. Further studies combing activation paradigms, tonotopic mapping and tract tracing, preferably with 7T MRI, are needed to address the issue of the number of core areas and of their organization.

The intrinsic connectivity, which has been traced in post-mortem material with DiI, is compatible with hierarchically distinct tiers among the early-stage auditory areas. Intrinsic connections were shown to originate mostly from layer II-III pyramidal neurons and to spread, at short distances, over all cortical layers. They involved a narrow part of the adjacent cortex within PAC and a larger part within the belt areas, where they tended to have anisotropic distributions (Tardif and Clarke 2001). The observed differences suggest that PAC relies on close collaboration between adjacent units or modules, whereas the belt areas play a more important role in the integration of different auditory features. 
Auditory processing streams

The three-tier architecture of human auditory areas and the cascade-like pattern of their connections beyond the parabelt areas speak in favor of a hierarchical organization of auditory processing; it provides support for computational models of serial processing, such as proposed for sound object recognition on the basis of dynamic causal modeling of fMRI data (Kumar et al. 2007). Within the six architectonically distinct areas of the belt, four types of functional specialization were demonstrated in previous studies; they may be at the origin of four parallel networks. One network is likely to be dedicated to speech processing as indicated by the corresponding specialization of areas LA and STA (Scott and Johnsrude 2003). The three other networks may be involved in sound recognition, sound localization or the combination of both (Altmann et al. 2007; Arnott et al. 2004; Griffiths and Warren 2002; Hart et al. 2004; Hunter et al. 2003; Viceic et al. 2006). Comparison with architectonically identified areas and activation patterns has shown that areas AA and ALA were activated by sound recognition, but not or significantly less by sound localization, whereas areas LA, STA and PA (Viceic et al. 2006), and more generally the planum temporale (Griffiths and Warren 2002) were involved in both. Of the two areas which are selective for sound recognition and may thus belong to a semantic network, ALA, but not AA, was shown to carry additional spatial information. The location corresponding to ALA (i.e., the lateral part of Heschl's gyrus), but not the more anterior planum polare where AA is located, was found to respond to pitch and to interaural correlation; the latter is a space-like feature, which does not, however, allow explicit localization of sounds (Budd et al. 2003). A recent $7 \mathrm{~T}$ study showed that activation by environmental sounds was modulated by positional information in ALA, but not in AA (van der Zwaag et al. 2011).

The connectivity pattern of the core, belt and parabelt regions suggests that there is a certain amount of cross-talk between the putative networks. Within the belt, adjacent areas are strongly connected; the communication between streams is further amplified by the divergent belt-parabelt connections and by the rather wide-spread connections within the parabelt. Within the cortical regions known to be involved in auditory processing, the optimal community structure identified two major modules, one centered on the temporal and the other on the parietal convexity (Fig. 3), which may represent the structural basis for the ventral and dorsal auditory processing streams, which have been shown to be involved in sound recognition and sound localization, respectively (Kaas and Hackett 2000; Rauschecker and Tian 2000; Rauschecker and Scott 2009; Rama et al. 2004; Krumbholz et al. 2007; Latinus et al. 2013).
The existence of distinct rostral and caudal fiber pathways was demonstrated using DTI. Functional MRI during listening to short sentences was used to determine effective connectivity between the PAC and non-primary auditory cortex (Upadhyay et al. 2008). Two regions of effective connectivity were identified, one on the lateral planum polare and the anterior part of the superior temporal gyrus and the other on the lateral planum temporal and the posterior part of the superior temporal gyrus; DTI identified distinct fiber tracts from PAC to each of the two regions.

Several lines of evidence speak in favor of a strong similarity in the organization of the auditory cortex in nonhuman primates and in man. In both, there is a strong evidence for architectonically heterogeneous early-stage areas (Morosan et al. 2001, 2005; Rivier and Clarke 1997; Clarke and Rivier 1998; Wallace et al. 2002; Kaas and Hackett 2000; Rauschecker and Tian 2000; Chiry et al. 2003), with putative functional specialization (Tian et al. 2001; Petkov et al. 2006); for human studies see above) and a three-tier connectivity pattern (Kaas and Hackett 2000) here. As in man, in non-human primates, auditory processing involves large parts of the temporo-parieto-prefrontal convexity, beyond the early-stage areas (Poremba and Mishkin 2007). There is a growing evidence for hemispheric asymmetry in processing species-specific calls in non-human primates (Poremba et al. 2004; Gil-da-Costa et al. 2006; Gil-da-Costa and Hauser 2006). However, to our knowledge there is no evidence for asymmetry in cortical connectivity in non-human primates. Furthermore, connections between the posterior temporal and the frontal areas are sparser in non-human primates than in man. Although anterograde and retrograde tracing techniques clearly demonstrated reciprocal connections between macaque belt and frontal areas (AL and CL to areas 8, 10 and 46; Romanski et al. 1999), the involved arcuate fasciculus (Schmahmann and Pandya 2006; Schmahmann et al. 2007) is less developed in non-human primates than in man (Thiebaut de Schotten et al. 2012). The latter has been repeatedly demonstrated in in vivo tracing studies (Kier et al. 2004; Catani et al. 2005; Parker et al. 2005; Powell et al. 2006; Glasser and Rilling 2008). Recent results from electrical recordings in chronically implanted patients indicate that this pathway is robust and rapidly conducting (Garell et al. 2013).

Hemispheric asymmetry in auditory cortico-cortical connectivity

The asymmetry which we found in respect to regional connectivity could be related, at least partially, to hemispheric specialization for language and for spatial functions. The posterior part of the superior temporal gyrus, 
the angular and supramarginal gyri, and pars opercularis of the inferior frontal gyrus, which displayed stronger connectivity on the left side, are known to be involved in speech processing (Hickok and Poeppel 2007; Friederici et al. 2009; Saur et al. 2010) and the specific connections which we found stronger are likely to be part of language pathways, which were shown in previous DTI studies to be larger on the left side (Barrick et al. 2007; Gharabaghi et al. 2009; Hagmann et al. 2006; Parker et al. 2005; Thiebaut de Schotten et al. 2011; Powell et al. 2006). It is, however, interesting to note that the posterior part of the superior temporal gyrus appears to be asymmetric in a more complex way. A post-mortem tracing study revealed a patchy pattern of intrinsic connections within this region; the spacing between intrinsically connected patches was wider on the left than on the right side, suggesting differences in intrinsic organization (Galuske et al. 2000). Our finding that the interareal connectivity of this region is stronger on the left side further supports differences in intrinsic organization, with a more integrative role on the left side.

The anterior part of the middle temporal gyrus, the inferior parietal gyrus, and pars triangularis of the inferior frontal gyrus, which displayed stronger connectivity on the right side, belong to regions known to be part of the right hemispheric network for auditory spatial processing (e.g., Spierer et al. 2009; At et al. 2011). The temporo-parietal contingent of these connections may contribute to the white matter pathway between the temporal lobe and the superior parietal lobule, which was found to be larger on the right side in a previous DTI study (Barrick et al. 2007).

\section{Conclusions}

The connectivity of the human auditory cortex revealed a three-tier architecture within the early-stage auditory areas, with a strong degree of integration and segregation. At this level, in vivo DSI tractography revealed an architecture that bears strong similarity to non-human primates. Putative higher-order auditory areas on the temporal and parietal convexities had a more widely spread local connections as well as long-range connections to the prefrontal cortex. Unlike in non-human primates, the connectivity pattern of higher-order auditory areas presented hemispheric asymmetry, which may represent the structural basis for the lateralization of cognitive functions.

Acknowledgments This work has been supported by the Swiss National Science Foundation Grants 3200030-124897 and 320030B141177 to S. Clarke. PH was supported by Leenaards Foundation.

\section{References}

Altmann CF, Bledowski C, Wibral M, Kaiser J (2007) Processing of location and pattern changes of natural sounds in the human auditory cortex. Neuroimage 35(3):1192-1200

Arnott SR, Binns MA, Grady CL, Alain C (2004) Assessing the auditory dual-pathway model in humans. Neuroimage 22(1):401-408

At A, Spierer L, Clarke S (2011) The role of the right parietal cortex in sound localization: a chronometric single pulse transcranial magnetic stimulation study. Neuropsychologia 49(9):2794-2797

Barrick TR, Lawes IN, Mackay CE, Clark CA (2007) White matter pathway asymmetry underlies functional lateralization. Cereb Cortex 17(3):591-598

Belin P (2006) Voice processing in human and non-human primates. Philos Trans R Soc Lond B Biol Sci 361(1476):2091-2107. doi:10.1098/rstb.2006.1933

Bellmann A, Meuli R, Clarke S (2001) Two types of auditory neglect. Brain 124(Pt 4):676-687

Brodmann K (1909) Vergleichende localisationslehre der grosshirnrinde in ihren prinzipien dargestellt auf grund des zellenbaues. Johann Ambrosius Barth, Leipzig

Brugge JF, Volkov IO, Garell PC, Reale RA, Howard MA 3rd (2003) Functional connections between auditory cortex on Heschl's gyrus and on the lateral superior temporal gyrus in humans. J Neurophysiol 90(6):3750-3763. doi:10.1152/jn.00500.2003

Brugge JF, Volkov IO, Oya H, Kawasaki H, Reale RA, Fenoy A, Steinschneider M, Howard MA 3rd (2008) Functional localization of auditory cortical fields of human: click-train stimulation. Hear Res 238(1-2):12-24. doi:10.1016/j.heares.2007.11.012

Budd TW, Hall DA, Goncalves MS, Akeroyd MA, Foster JR, Palmer AR, Head K, Summerfield AQ (2003) Binaural specialisation in human auditory cortex: an fMRI investigation of interaural correlation sensitivity. Neuroimage 20(3):1783-1794

Bullmore E, Sporns O (2009) Complex brain networks: graph theoretical analysis of structural and functional systems. Nat Rev Neurosci 10(3):186-198

Bullmore E, Sporns O (2012) The economy of brain network organization. Nat Rev Neurosci 13(5):336-349. doi:10.1038/ nrn3214

Burgel U, Amunts K, Hoemke L, Mohlberg H, Gilsbach JM, Zilles K (2006) White matter fiber tracts of the human brain: threedimensional mapping at microscopic resolution, topography and intersubject variability. Neuroimage 29(4):1092-1105. doi:10. 1016/j.neuroimage.2005.08.040

Bushara KO, Weeks RA, Ishii K, Catalan MJ, Tian B, Rauschecker JP, Hallett M (1999) Modality-specific frontal and parietal areas for auditory and visual spatial localization in humans. Nat Neurosci 2(8):759-766

Cammoun L, Gigandet X, Meskaldji D, Thiran JP, Sporns O, Do KQ, Maeder P, Meuli R, Hagmann P (2012) Mapping the human connectome at multiple scales with diffusion spectrum MRI. J Neurosci Methods 203(2):386-397. doi:10.1016/j.jneumeth. 2011.09.031

Catani M, Howard RJ, Pajevic S, Jones DK (2002) Virtual in vivo interactive dissection of white matter fasciculi in the human brain. Neuroimage 17(1):77-94

Catani M, Jones DK, Ffytche DH (2005) Perisylvian language networks of the human brain. Ann Neurol 57(1):8-16. doi:10. 1002/ana.20319

Chiry O, Tardif E, Magistretti PJ, Clarke S (2003) Patterns of calcium-binding proteins support parallel and hierarchical organization of human auditory areas. Eur J Neurosci 17(2):397-410 
Clarke S (1994) Association and intrinsic connections of human extrastriate visual cortex. Proc Biol Sci Roy Soc 257(1348):87-92

Clarke S, Miklossy J (1990) Occipital cortex in man: organization of callosal connections, related myelo- and cytoarchitecture, and putative boundaries of functional visual areas. J Comp Neurol 298(2): 188-214

Clarke S, Rivier F (1998) Compartments within human primary auditory cortex: evidence from cytochrome oxidase and acetylcholinesterase staining. Eur J Neurosci 10:741-745

Clarke S, Riahi-Arya S, Tardif E, Eskenasy AC, Probst A (1999) Thalamic projections of the fusiform gyrus in man. Eur $\mathbf{J}$ Neurosci 11(5):1835-1838

Da Costa S, van der Zwaag W, Marques JP, Frackowiak RS, Clarke S, Saenz M (2011) Human primary auditory cortex follows the shape of Heschl's gyrus. J Neurosci 31(40):14067-14075

de la Mothe LA, Blumell S, Kajikawa Y, Hackett TA (2006) Cortical connections of the auditory cortex in marmoset monkeys: core and medial belt regions. J Comp Neurol 496(1):27-71

Desikan RS, Segonne F, Fischl B, Quinn BT, Dickerson BC, Blacker D, Buckner RL, Dale AM, Maguire RP, Hyman BT, Albert MS, Killiany RJ (2006) An automated labeling system for subdividing the human cerebral cortex on MRI scans into gyral based regions of interest. NeuroImage 31(3):968-980

Di Virgilio G, Clarke S (1997) Direct interhemispheric visual input to human speech areas. Hum Brain Mapp 5(5):347-354

Di Virgilio G, Clarke S, Pizzolato G, Schaffner T (1999) Cortical regions contributing to the anterior commissure in man. Exp Brain Res 124(1):1-7

Ding SL, Van Hoesen GW, Cassell MD, Poremba A (2009) Parcellation of human temporal polar cortex: a combined analysis of multiple cytoarchitectonic, chemoarchitectonic, and pathological markers. J Comp Neurol 514(6):595-623. doi:10. 1002/cne. 22053

Fischl B, van der Kouwe A, Destrieux C, Halgren E, Segonne F, Salat DH, Busa E, Seidman LJ, Goldstein J, Kennedy D, Caviness V, Makris N, Rosen B, Dale AM (2004) Automatically parcellating the human cerebral cortex. Cereb Cortex 14(1):11-22

Friederici AD, Makuuchi M, Bahlmann J (2009) The role of the posterior superior temporal cortex in sentence comprehension. NeuroReport 20(6):563-568

Galaburda A, Sanides F (1980) Cytoarchitectonic organization of the human auditory cortex. J Comp Neurol 190(3):597-610

Galuske RA, Schlote W, Bratzke H, Singer W (2000) Interhemispheric asymmetries of the modular structure in human temporal cortex. Science 289(5486):1946-1949

Garell PC, Bakken H, Greenlee JD, Volkov I, Reale RA, Oya H, Kawasaki H, Howard MA, Brugge JF (2013) Functional connection between posterior superior temporal gyrus and ventrolateral prefrontal cortex in human. Cereb Cortex 23(10):2309-2321. doi:10.1093/cercor/bhs220

Gharabaghi A, Kunath F, Erb M, Saur R, Heckl S, Tatagiba M, Grodd W, Karnath HO (2009) Perisylvian white matter connectivity in the human right hemisphere. BMC Neurosci 10:15

Gigandet X (2009) Global brain connectivity analysis by diffusion MR tractography: algorithms, validation and applications. Doctoral Thesis. EPFL, Institute of Electrical Engineering, Lausanne

Gil-da-Costa R, Hauser MD (2006) Vervet monkeys and humans show brain asymmetries for processing conspecific vocalizations, but with opposite patterns of laterality. Proc Biol Sci Roy Soc 273(1599):2313-2318. doi:10.1098/rspb.2006.3580

Gil-da-Costa R, Martin A, Lopes MA, Munoz M, Fritz JB, Braun AR (2006) Species-specific calls activate homologs of Broca's and Wernicke's areas in the macaque. Nat Neurosci 9(8):1064-1070. doi: $10.1038 / \mathrm{nn} 1741$
Glasser MF, Rilling JK (2008) DTI tractography of the human brain's language pathways. Cereb Cortex 18(11):2471-2482. doi:10. 1093/cercor/bhn011

Gong G, He Y, Concha L, Lebel C, Gross DW, Evans AC, Beaulieu C (2009) Mapping anatomical connectivity patterns of human cerebral cortex using in vivo diffusion tensor imaging tractography. Cereb Cortex 19(3):524-536

Gourevitch B, Le Bouquin Jeannes R, Faucon G, Liegeois-Chauvel C (2008) Temporal envelope processing in the human auditory cortex: response and interconnections of auditory cortical areas. Hear Res 237(1-2):1-18. doi:10.1016/j.heares.2007.12.003

Griffiths TD, Warren JD (2002) The planum temporale as a computational hub. Trends Neurosci 25(7):348-353

Gueguin M, Le Bouquin-Jeannes R, Faucon G, Chauvel P, LiegeoisChauvel C (2007) Evidence of functional connectivity between auditory cortical areas revealed by amplitude modulation sound processing. Cereb Cortex 17(2):304-313. doi:10.1093/cercor/ bhj148

Hackett TA, Stepniewska I, Kaas JH (1998) Subdivisions of auditory cortex and ipsilateral cortical connections of the parabelt auditory cortex in macaque monkeys. J Comp Neurol 394(4):475-495

Hackett TA, Stepniewska I, Kaas JH (1999) Prefrontal connections of the parabelt auditory cortex in macaque monkeys. Brain Res 817(1-2):45-58

Hackett TA, Smiley JF, Ulbert I, Karmos G, Lakatos P, de la Mothe LA, Schroeder CE (2007) Sources of somatosensory input to the caudal belt areas of auditory cortex. Perception 36(10):1419-1430

Hagmann P, Cammoun L, Martuzzi R, Maeder P, Clarke S, Thiran JP, Meuli R (2006) Hand preference and sex shape the architecture of language networks. Hum Brain Mapp 27(10):828-835

Hagmann P, Kurant M, Gigandet X, Thiran P, Wedeen VJ, Meuli R, Thiran JP (2007) Mapping human whole-brain structural networks with diffusion MRI. PLoS ONE 2(7):e597

Hagmann P, Cammoun L, Gigandet X, Meuli R, Honey CJ, Wedeen VJ, Sporns O (2008) Mapping the structural core of human cerebral cortex. PLoS Biol 6(7):e159

Hart HC, Palmer AR, Hall DA (2004) Different areas of human nonprimary auditory cortex are activated by sounds with spatial and nonspatial properties. Hum Brain Mapp 21(3):178-190

Hickok G, Poeppel D (2007) The cortical organization of speech processing. Nat Rev Neurosci 8(5):393-402

Hochberg Y, Benjamini Y (1990) More powerful procedures for multiple significance testing. Stat Med 9(7):811-818

Howard MA, Volkov IO, Mirsky R, Garell PC, Noh MD, Granner M, Damasio H, Steinschneider M, Reale RA, Hind JE, Brugge JF (2000) Auditory cortex on the human posterior superior temporal gyrus. J Comp Neurol 416(1):79-92

Humphries C, Liebenthal E, Binder JR (2010) Tonotopic organization of human auditory cortex. Neuroimage 50(3):1202-1211

Hunter MD, Smith JK, Taylor N, Woods W, Spence SA, Griffiths TD, Woodruff PW (2003) Laterality effects in perceived spatial location of hallucination-like voices. Percept Mot Skills 97(1):246-250

Kaas JH, Hackett TA (2000) Subdivisions of auditory cortex and processing streams in primates. Proc Natl Acad Sci USA 97(22):11793-11799. doi:10.1073/pnas.97.22.11793

Kaas JH, Hackett TA, Tramo MJ (1999) Auditory processing in primate cerebral cortex. Curr Opin Neurobiol 9(2):164-170

Kier EL, Staib LH, Davis LM, Bronen RA (2004) Anatomic dissection tractography: a new method for precise MR localization of white matter tracts. AJNR Am J Neuroradiol 25(5):670-676

Krumbholz K, Eickhoff SB, Fink GR (2007) Feature- and objectbased attentional modulation in the human auditory "where" 
pathway. J Cogn Neurosci 19(10):1721-1733. doi:10.1162/jocn. 2007.19.10.1721

Kumar S, Stephan KE, Warren JD, Friston KJ, Griffiths TD (2007) Hierarchical processing of auditory objects in humans. PLoS Comput Biol 3(6):e100

Langers DR (2014) Assessment of tonotopically organised subdivisions in human auditory cortex using volumetric and surfacebased cortical alignments. Hum Brain Mapp 35(4):1544-1561. doi: $10.1002 / \mathrm{hbm} .22272$

Latinus M, McAleer P, Bestelmeyer PE, Belin P (2013) Norm-based coding of voice identity in human auditory cortex. Current Biol 23(12):1075-1080. doi:10.1016/j.cub.2013.04.055

Lewis JW, Wightman FL, Brefczynski JA, Phinney RE, Binder JR, DeYoe EA (2004) Human brain regions involved in recognizing environmental sounds. Cereb Cortex 14(9):1008-1021

Liegeois-Chauvel C, Musolino A, Chauvel P (1991) Localization of the primary auditory area in man. Brain 114(Pt 1A):139-151

Liegeois-Chauvel C, Musolino A, Badier JM, Marquis P, Chauvel P (1994) Evoked potentials recorded from the auditory cortex in man: evaluation and topography of the middle latency components. Electroencephalogr Clin Neurophysiol 92(3):204-214

Maeder PP, Meuli RA, Adriani M, Bellmann A, Fornari E, Thiran JP, Pittet A, Clarke S (2001) Distinct pathways involved in sound recognition and localization: a human fMRI study. Neuroimage 14(4):802-816

Maslov S, Sneppen K (2002) Specificity and stability in topology of protein networks. Science 296(5569):910-913

Miklossy J, Clarke S, Van der Loos H (1991) The long distance effects of brain lesions: visualization of axonal pathways and their terminations in the human brain by the Nauta method. J Neuropathol Exp Neurol 50(5):595-614

Mones E, Vicsek L, Vicsek T (2012) Hierarchy measure for complex networks. PLoS ONE 7(3):e33799

Morel A, Garraghty PE, Kaas JH (1993) Tonotopic organization, architectonic fields, and connections of auditory cortex in macaque monkeys. J Comp Neurol 335(3):437-459

Morosan P, Rademacher J, Schleicher A, Amunts K, Schormann T, Zilles K (2001) Human primary auditory cortex: cytoarchitectonic subdivisions and mapping into a spatial reference system. Neuroimage 13(4):684-701

Morosan P, Schleicher A, Amunts K, Zilles K (2005) Multimodal architectonic mapping of human superior temporal gyrus. Anat Embryol (Berl) 210(5-6):401-406

Newman ME (2006) Modularity and community structure in networks. Proc Natl Acad Sci USA 103(23):8577-8582

Pandya DN, Rosene DL, Doolittle AM (1994) Corticothalamic connections of auditory-related areas of the temporal lobe in the rhesus monkey. J Comp Neurol 345(3):447-471. doi:10.1002/ cne. 903450311

Parker GJ, Luzzi S, Alexander DC, Wheeler-Kingshott CA, Ciccarelli O, Lambon Ralph MA (2005) Lateralization of ventral and dorsal auditory-language pathways in the human brain. Neuroimage 24(3):656-666

Petkov CI, Kayser C, Augath M, Logothetis NK (2006) Functional imaging reveals numerous fields in the monkey auditory cortex. PLoS Biol 4(7):e215. doi:10.1371/journal.pbio.0040215

Poremba A, Mishkin M (2007) Exploring the extent and function of higher-order auditory cortex in rhesus monkeys. Hear Res 229(1-2):14-23. doi:10.1016/j.heares.2007.01.003

Poremba A, Malloy M, Saunders RC, Carson RE, Herscovitch P, Mishkin M (2004) Species-specific calls evoke asymmetric activity in the monkey's temporal poles. Nature 427(6973):448-451. doi:10.1038/nature02268

Powell HW, Parker GJ, Alexander DC, Symms MR, Boulby PA, Wheeler-Kingshott CA, Barker GJ, Noppeney U, Koepp MJ, Duncan JS (2006) Hemispheric asymmetries in language-related pathways: a combined functional MRI and tractography study. NeuroImage 32(1):388-399. doi:10.1016/j.neuroimage.2006.03. 011

Rademacher J, Morosan P, Schormann T, Schleicher A, Werner C, Freund HJ, Zilles K (2001) Probabilistic mapping and volume measurement of human primary auditory cortex. Neuroimage 13(4):669-683

Rama P, Poremba A, Sala JB, Yee L, Malloy M, Mishkin M, Courtney SM (2004) Dissociable functional cortical topographies for working memory maintenance of voice identity and location. Cereb Cortex 14(7):768-780. doi:10.1093/cercor/ bhh037

Rauschecker JP, Scott SK (2009) Maps and streams in the auditory cortex: nonhuman primates illuminate human speech processing. Nat Neurosci 12(6):718-724

Rauschecker JP, Tian B (2000) Mechanisms and streams for processing of "what" and "where" in auditory cortex. Proc Natl Acad Sci USA 97(22):11800-11806. doi:10.1073/pnas.97. 22.11800

Rivier F, Clarke S (1997) Cytochrome oxidase, acetylcholinesterase, and NADPH-diaphorase staining in human supratemporal and insular cortex: evidence for multiple auditory areas. Neuroimage 6(4):288-304

Romanski LM, Tian B, Fritz J, Mishkin M, Goldman-Rakic PS, Rauschecker JP (1999) Dual streams of auditory afferents target multiple domains in the primate prefrontal cortex. Nat Neurosci 2(12):1131-1136. doi:10.1038/16056

Rose JE, Woolsey CN (1949) The relations of thalamic connections, cellular structure and evocable electrical activity in the auditory region of the cat. J Comp Neurol 91(3):441-466

Saenz M, Langers DR (2014) Tonotopic mapping of human auditory cortex. Hear Res 307:42-52. doi:10.1016/j.heares. 2013.07.016

Saur D, Schelter B, Schnell S, Kratochvil D, Kupper H, Kellmeyer P, Kummerer D, Kloppel S, Glauche V, Lange R, Mader W, Feess D, Timmer J, Weiller C (2010) Combining functional and anatomical connectivity reveals brain networks for auditory language comprehension. NeuroImage 49(4):3187-3197

Schmahmann JD, Pandya DN (2006) Fiber pathways of the brain, vol Pt 3. New York

Schmahmann JD, Pandya DN, Wang R, Dai G, D'Arceuil HE, de Crespigny AJ, Wedeen VJ (2007) Association fibre pathways of the brain: parallel observations from diffusion spectrum imaging and autoradiography. Brain 130(Pt 3):630-653

Schonwiesner M, von Cramon DY, Rubsamen R (2002) Is it tonotopy after all? NeuroImage 17(3):1144-1161

Scott SK, Johnsrude IS (2003) The neuroanatomical and functional organization of speech perception. Trends Neurosci 26(2):100-107

Smiley JF, Hackett TA, Ulbert I, Karmas G, Lakatos P, Javitt DC, Schroeder CE (2007) Multisensory convergence in auditory cortex, I. Cortical connections of the caudal superior temporal plane in macaque monkeys. J Comp Neurol 502(6):894-923

Spierer L, Bellmann-Thiran A, Maeder P, Murray MM, Clarke S (2009) Hemispheric competence for auditory spatial representation. Brain 132:1963-1966

Sporns O (2011) From simple graphs to the connectome: networks in neuroimaging. NeuroImage 62(2):881-886

Talavage TM, Sereno MI, Melcher JR, Ledden PJ, Rosen BR, Dale AM (2004) Tonotopic organization in human auditory cortex revealed by progressions of frequency sensitivity. J Neurophysiol 91(3):1282-1296

Tardif E, Clarke S (2001) Intrinsic connectivity of human auditory areas: a tracing study with DiI. Eur J Neurosci 13(5):1045-1050

Tardif E, Spierer L, Clarke S, Murray MM (2008) Interactions between auditory 'what' and 'where' pathways revealed by 
enhanced near-threshold discrimination of frequency and position. Neuropsychologia 46(4):958-966

Thiebaut de Schotten M, Ffytche DH, Bizzi A, Dell'Acqua F, Allin M, Walshe M, Murray R, Williams SC, Murphy DG, Catani M (2011) Atlasing location, asymmetry and inter-subject variability of white matter tracts in the human brain with MR diffusion tractography. NeuroImage 54(1):49-59. doi:10.1016/j.neuro image.2010.07.055

Thiebaut de Schotten M, Dell'Acqua F, Valabregue R, Catani M (2012) Monkey to human comparative anatomy of the frontal lobe association tracts. Cortex 48(1):82-96. doi:10.1016/j.cortex. 2011.10.001

Thiran AB, Clarke S (2003) Preserved use of spatial cues for sound segregation in a case of spatial deafness. Neuropsychologia 41(9):1254-1261

Tian B, Reser D, Durham A, Kustov A, Rauschecker JP (2001) Functional specialization in rhesus monkey auditory cortex. Science 292(5515):290-293. doi:10.1126/science.1058911

Upadhyay J, Ducros M, Knaus TA, Lindgren KA, Silver A, TagerFlusberg H, Kim DS (2007) Function and connectivity in human primary auditory cortex: a combined fMRI and DTI study at 3 Tesla. Cereb Cortex 17(10):2420-2432

Upadhyay J, Silver A, Knaus TA, Lindgren KA, Ducros M, Kim DS, Tager-Flusberg H (2008) Effective and structural connectivity in the human auditory cortex. J Neurosci 28(13):3341-3349. doi:10.1523/JNEUROSCI.4434-07.2008

van der Zwaag W, Gentile G, Gruetter R, Spierer L, Clarke S (2011) Where sound position influences sound object representations: a 7-T fMRI study. Neuroimage 54(3):1803-1811
Viceic D, Fornari E, Thiran JP, Maeder PP, Meuli R, Adriani M, Clarke S (2006) Human auditory belt areas specialized in sound recognition: a functional magnetic resonance imaging study. Neuroreport 17(16):1659-1662

Viceic D, Campos R, Fornari E, Spierer L, Meuli R, Clarke S, Thiran JP (2009) Local landmark-based registration for fMRI group studies of nonprimary auditory cortex. Neuroimage 44(1):145-153. doi:10.1016/j.neuroimage.2008.07.051

von Economo C, Horn L (1930) Über Windungsrelief, Maße und Rindenarchitektonik der Supratemporalfläche, ihre individuellen und ihre Seitenunterschiede. Zeitschrift für die gesamte Neurologie und Psychiatrie 130(1):678-757

Wallace MN, Johnston PW, Palmer AR (2002) Histochemical identification of cortical areas in the auditory region of the human brain. Exp Brain Res 143(4):499-508

Watts DJ, Strogatz SH (1998) Collective dynamics of 'small-world' networks. Nature 393(6684):440-442

Wedeen VJ, Hagmann P, Tseng WY, Reese TG, Weisskoff RM (2005) Mapping complex tissue architecture with diffusion spectrum magnetic resonance imaging. Magn Reson Med 54(6): 1377-1386

Wedeen VJ, Wang RP, Schmahmann JD, Benner T, Tseng WY, Dai G, Pandya DN, Hagmann P, D'Arceuil H, de Crespigny AJ (2008) Diffusion spectrum magnetic resonance imaging (DSI) tractography of crossing fibers. Neuroimage 41(4):1267-1277

Wiesendanger E, Clarke S, Kraftsik R, Tardif E (2004) Topography of cortico-striatal connections in man: anatomical evidence for parallel organization. Eur J Neurosci 20(7):1915-1922 